\title{
EGCG induces lung cancer A549 cell apoptosis by regulating $\mathrm{Ku} 70$ acetylation
}

\author{
MIN LI，JING-JING LI，QI-HUA GU，JIAN AN，LI-MING CAO，HUA-PING YANG and CHENG-PING HU \\ Department of Respiratory Medicine, Xiangya Hospital, Central South University, Changsha, Hunan 410008, P.R. China
}

Received November 4, 2015; Accepted December 12, 2015

DOI: $10.3892 /$ or.2016.4587

\begin{abstract}
Lung cancer is the leading cause of cancer-related death worldwide. (-)-Epigallocatechin-3-gallate (EGCG) is a potential chemopreventive and therapeutic agent for lung cancer. Induction of apoptosis was examined using Annexin V/PI double staining flow cytometry. Western blot analysis detected the protein expression of cleaved caspase-3, Bax and Bcl-xL. Co-immunoprecipitation was used to detect the interaction of Ku70-Bax and the acetylation status of Ku70. Treatment of A549 cells with EGCG-induced apoptosis via increased expression of cleaved caspase-3 and Bax, but decreased expression of Bcl-xL. EGCG upregulated the K70 acetylation status of A549 cells and downregulated the interaction of Bax-Ku70 in a concentration- and time-dependent manner. The apoptosis-promoting effect of EGCG on A549 cells was obviously weakened, along with strengthening of the Bax-Ku70 interaction, after pCDNA3.1(+)-Ku70 plasmid and pCDNA3.1(+)-Ku70 ${ }^{539 / 542 \mathrm{R}}$ plasmid transfection. Our results established a role of EGCG in inducing cell apoptosis by suppressing Bax activity. Regulating Ku70 acetylation by EGCG, that block the interaction between Ku70 and Bax, will result in lung cancer cell apoptosis.
\end{abstract}

\section{Introduction}

Lung cancer is one of the malignant tumors with very high global incidence and mortality (1). In addition to smoking cessation and early detection measures, chemoprevention has become an important means of prevention and control of lung cancer (2). Green tea is widely consumed for its characteristic flavor and potential health benefits. Many studies have provided evidence that green tea and its components reduce the risk of cancers, including lung, prostate, and breast cancers (3-6).

Correspondence to: Professor Cheng-Ping Hu, Department of Respiratory Medicine, Xiangya Hospital, Central South University, Changsha, Hunan 410008, P.R. China

E-mail: huchengp28@126.com

Key words: apoptosis, Ku70 acetylation, Bax, (-)-Epigallocatechin3-gallate, tea, lung cancer, A549
A typical cup of green tea contains 100-150 mg of tea polyphenols; the major green tea polyphenol is (-)-epigallocatechin-3-gallate (EGCG), which comprises more than $50 \%$ of total tea polyphenols (7). Research has shown that EGCG is a potential chemopreventive and therapeutic agent for various tumors (8-10). EGCG has been demonstrated to act on multiple key elements in signal transduction pathways related to inhibition of carcinogen-induced mutagenesis $(11,12)$, induction of cell cycle arrest (13), induction of apoptosis (14), inhibition of growth factor-mediated proliferation (15), inhibition of transformation (9), inhibition of angiogenesis (16) and inhibition of telomerase activity (17). It has been shown that EGCG can effectively regulate various key molecules in cell mitochondrial apoptosis pathways in other tumors as a potential antitumor substance $(18,19)$. However, the molecular mechanisms of EGCG inducing apoptosis have not been completely elucidated in lung cancer.

$\mathrm{Ku} 70$ was first characterized as part of the $\mathrm{Ku} 70 / \mathrm{Ku} 80$ heterodimer that is essential as a DNA binding component of the non-homologous end joining (NHEJ) double-strand break (DSB) repair (20). Although Ku70 was originally found in the nucleus, its cytoplasmic function has been investigated as a regulatory factor of cell death through interaction with an apoptotic protein Bax (21). Dissociating Bax from Ku70, either by pharmacological means or by agents that block the interaction between Ku70 and Bax, may result in cell death (21). Our previous study revealed that EGCG could effectively inhibit the growth of lung adenocarcinoma A549 cell line transplanted tumors and that the general mechanism involved interference with the interaction between Ku70 and Bax (22). Therefore, the specific mechanism of the EGCG-regulated interaction of $\mathrm{Ku} 70-\mathrm{Bax}$ that induces apoptosis in lung cancer A549 cells is further explored in the present study.

\section{Materials and methods}

Cell lines, strains and plasmid vector. The human lung adenocarcinoma A549 cell line was purchased from the Cell Bank of Xiangya School of Medicine, Central South University. E. Coli DH5 $\alpha$ was provided by the Cancer Research Institute of Xiangya School of Medicine, Central South University. The pMD 18-T vector kit was purchased from Takara (Otsu, Japan). The pCDNA3.1 (+) plasmid was supplied by the Cancer Research Institute of Xiangya School of Medicine, Central South University. 
Drugs and reagents. EGCG (purity 98\%), dimethyl sulfoxide (DMSO) (both from Sigma, St. Louis, MO, USA), Annexin V and PI double staining flow method cell apoptosis detection kit (Invitrogen, Carlsbad, CA, USA), RT-PCR kit (two-step method), real-time PCR kit (both from Fermentas, Vilnius, Lithuania), rabbit anti-human Bcl-xL antibody (Proteintech, Chicago, IL, USA), rabbit anti-human Bax antibody (Cell Signaling Technology, Boston, MA, USA), rabbit anti-human caspase-3 antibody (Auragene, Changsha, China), and rabbit anti-human Ku70 antibody (Santa Cruz Biotechnology, Inc., Dallas, TX, USA).

Construction of gene point mutation plasmid. Ku70-pcDNA3.1 recombinant plasmid was used as the template. Two mutation primers were designed in the locus to be mutated (the primers were: Mut-Ku-F, AAGGGAGAGTTACCAGGAGAAAA CACGATAATGAAGGTTCTGGAA and Mut-Ku-R, TTCTC CTGGTAACTCTCCCTTCAGGATTGTAATCTGGTGGG TAAAC, respectively) for PCR amplification. The conditions of reaction system were pre-denaturation at $94^{\circ} \mathrm{C}$ for $4 \mathrm{~min}$, $94^{\circ} \mathrm{C}$ for $30 \mathrm{sec}, 56^{\circ} \mathrm{C}$ for $30 \mathrm{sec}$ and $72^{\circ} \mathrm{C}$ for $7 \mathrm{~min}$, for a total of 30 amplification cycles. The Notch mutant was amplified. A total of $1 \mu \mathrm{l}$ of $D p n \mathrm{I}$ restriction endonuclease was added to the thermal cycling product. The template plasmid without mutation was digested. A total of $5 \mu 1$ of digested thermal cycle product was used to transform competent bacteria. Then, $5 \mathrm{ml}$ of bacteria were agitated overnight. The plasmid was extracted from the bacterial suspension. The nucleic acid electrophoresis confirmed plasmid bands. A small amount of bacterial suspension was used for plasmid extraction and sequencing. The residual bacterial suspension was frozen.

Apoptosis analysis. The cells in each group were treated with different concentrations of EGCG for given times. Thereafter, the cells were divided into two groups; one was used for detecting the effect of EGCG on human lung cancer A549 cell survival rates in vitro by the MTT method, whereas the other was used for detecting cell apoptosis by Annexin V/PI double staining flow cytometry. The effects of EGCG with different concentrations and different action times on cell apoptosis in each group were comprehensively analyzed.

Western blotting. To detect protein expression in the cells of each group, total protein was extracted from the treated cells. The protein concentration was measured, and western blot analysis was conducted to analyze the expression of various proteins following the manufacturer's instructions. Aliquots of equal amounts of protein $(40 \mu \mathrm{g})$ from the cell lysates were subjected to SDS-PAGE electrophoresis and transferred to polyvinylidene difluoride (PVDF) membranes. After blocking the non-specific binding sites, the membranes were incubated overnight with the desired primary antibody at $4^{\circ} \mathrm{C}$. The membranes were then incubated with the respective HRP-conjugated secondary antibody for $1 \mathrm{~h}$ at room temperature and the immunoreactive bands were detected by enhanced chemiluminescence detection systems (Thermo Scientific, Waltham, MA, USA).

Co-immunoprecipitation. To detect the interaction of $\mathrm{Ku} 70-\mathrm{Bax}$ and the acetylation status of Ku70, the treated

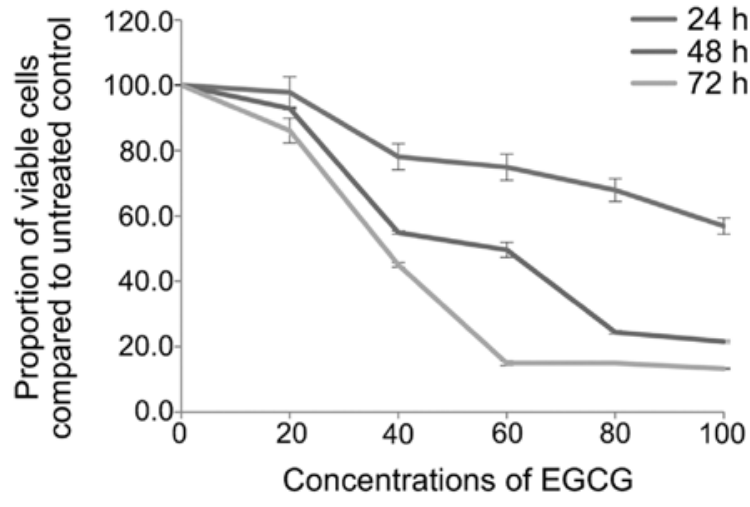

Figure 1. A549 cell survival rates when treated with EGCG (survival rates, \%; EGCG concentrations, $\mu \mathrm{mol} / \mathrm{l}$; effect time, h). The cell survival rate showed a declining trend along with the increase of EGCG concentration, displaying a significant dosage-effect relationship. The inhibitory rate in the 40 and $80 \mu \mathrm{mol} / 1$ dosage groups showed an increasing trend with prolongation of EGCG action time, displaying an time-effect relationship.

cell protein was extracted. The protein concentration was determined. A total of $1,000 \mu \mathrm{g}$ of cell lysate was divided into two portions. One portion was $100 \mu \mathrm{g}$. An equal volume of 2X SDS sample buffer was added, mixed, degenerated at $100^{\circ} \mathrm{C}$ in boiling water for $10 \mathrm{~min}$ and centrifuged. A total of $50 \mu \mathrm{g}$ of supernatant was extracted. The protein level in whole cell lysates was analyzed by western blotting. The remaining portion of the cell lysate was $900 \mu \mathrm{g}$. A total of $5 \mu \mathrm{l}$ of protein A/G agarose beads and a given amount of antibody (1-2 $\mu \mathrm{g})$ were added. Lysis buffer was added to a final volume of $1 \mathrm{ml}$. The sample was fixed in a vertical mixer and slowly rotated for $3 \mathrm{~h}$. The beads were washed three times with $1 \mathrm{ml}$ of lysis buffer and centrifuged in a refrigerated centrifuge at 3,000 rpm at $4^{\circ} \mathrm{C}$ for $3 \mathrm{~min}$. The supernatant was absorbed after the final washing. A total of $50 \mu 1$ of $1 \mathrm{X}$ SDS sample buffer was added, mixed evenly, boiled at $100^{\circ} \mathrm{C}$ for $10 \mathrm{~min}$ and centrifuged. A $15-\mu 1$ sample was extracted. The interaction between the proteins was analyzed by western blotting.

Statistical analysis. All of the data were processed by the statistical software SPSS10.0. The data are shown as the mean and standard deviation (mean $\pm \mathrm{SD}$ ). Student's t-test was used for comparisons between the two groups. The SNK-q test was used for comparisons among multiple groups. $\mathrm{P}<0.05$ indicates statistical significance.

\section{Results}

EGCG induces A549 cell apoptosis. After treating A549 cells with different concentrations of EGCG $(0,20,40,60,80$ and $100 \mu \mathrm{mol} / \mathrm{l})$ for 24,48 and $72 \mathrm{~h}$, A549 cell survival rates were detected by the MTT assay (Fig. 1), and apoptosis was detected by Annexin V/PI double staining flow cytometry (Fig. 2). After treating A549 cells with $40 \mu \mathrm{mol} / \mathrm{l}$ EGCG for $24 \mathrm{~h}$, cell growth was significantly inhibited $(\mathrm{P}=0.017)$. The inhibition was stronger after $48 \mathrm{~h}(\mathrm{P}<0.01)$ and even stronger again after $72 \mathrm{~h}(\mathrm{P}<0.01)$. The inhibitory rate in the other dosage groups also showed an increasing trend with prolongation of drug action time, displaying an obvious time-effect relationship (pair comparison $\mathrm{P}<0.05$ ). Furthermore, the cell survival 

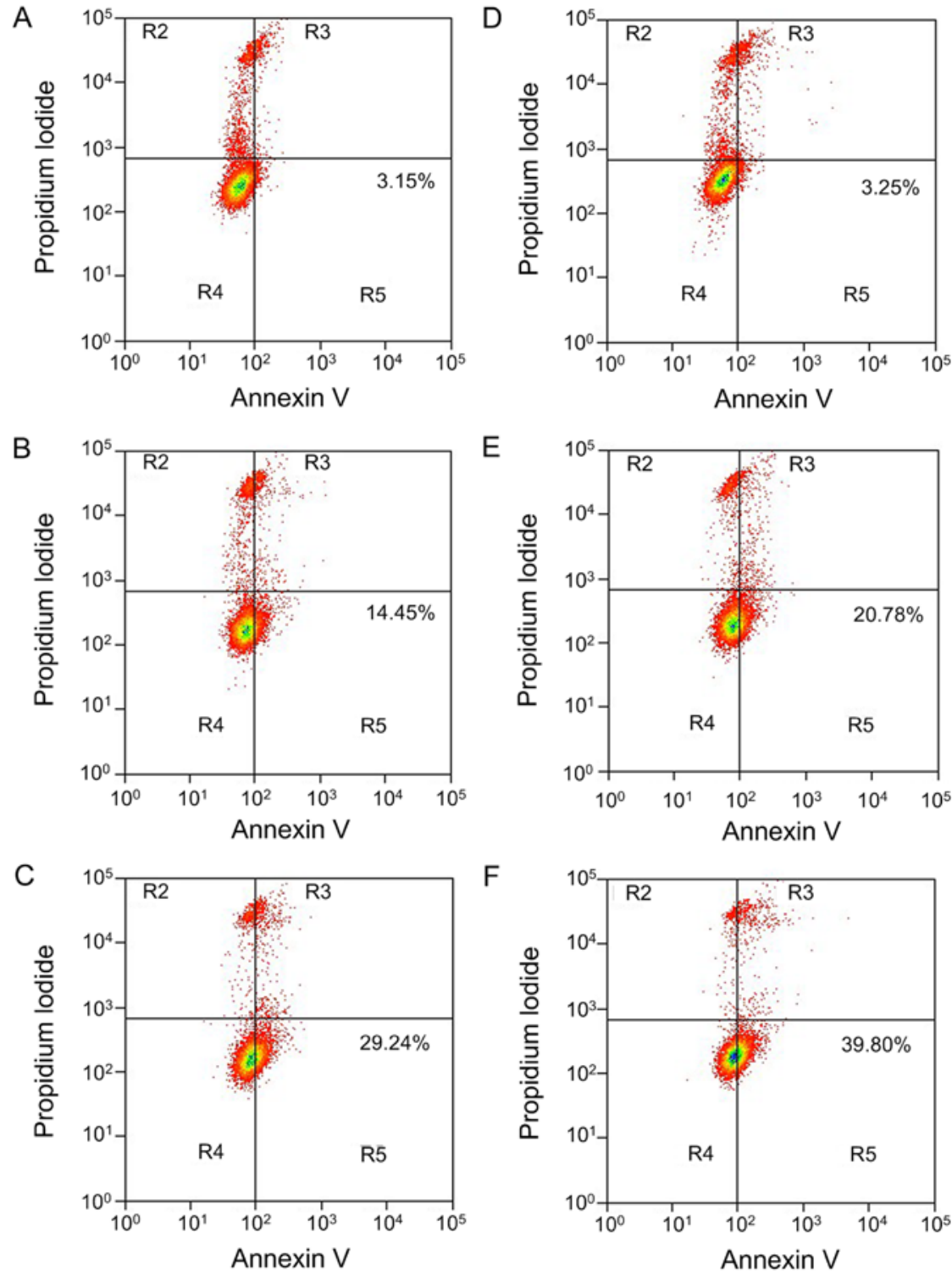

Figure 2. Apoptosis-inducing effect of A549 cells treated with EGCG, detected by Annexin V/PI double staining. (A) $0 \mu \mathrm{mol} / 1,24 \mathrm{~h}$; (B) $40 \mu \mathrm{mol} / 1,24 \mathrm{~h}$; (C) $80 \mu \mathrm{mol} / 1,24 \mathrm{~h}$; (D) $0 \mu \mathrm{mol} / 1,48 \mathrm{~h}$; (E) $40 \mu \mathrm{mol} / 1,48 \mathrm{~h}$; and (F) $80 \mu \mathrm{mol} / 1,48 \mathrm{~h}$. With the increase of EGCG concentration, the cell apoptosis rate in each group increased dose-dependently. The apoptosis rate in the 40 and $80 \mu \mathrm{mol} / 1$ dosage groups showed an increasing trend with prolongation of EGCG action time, displaying an time-effect relationship.

rate in each group also showed a declining trend along with the increase of EGCG concentration, displaying a significant dosage-effect relationship $(\mathrm{P}<0.05)$. In addition, with the increase of EGCG concentration, the early cell apoptosis rate in each group showed a increasing trend along with the increase of EGCG concentration, displaying a dose-dependent relationship $(\mathrm{P}<0.05)$. When the action time of EGCG was prolonged to $48 \mathrm{~h}$, the apoptosis rate also increased, and the apoptosis inducement ability showed a time-dependent relationship $(\mathrm{P}<0.05)$.

EGCG regulates cleaved caspase-3, Bax and Bcl-xL expression. After A549 cells were treated with EGCG concentrations of 0,40 and $80 \mu \mathrm{mol} / 1$ for 24 and $48 \mathrm{~h}$, western blot analysis was used to detect the protein expression of cleaved caspase-3 (Fig. 3), Bax (Fig. 4) and Bcl-xL (Fig. 5). The results showed that the protein expression of caspase-3 (17 kDa) and
Bax in the $0 \mu \mathrm{mol} / 1$ group was relatively low after $24 \mathrm{~h}$ of treatment. With an increase in EGCG concentration to 40 and $80 \mu \mathrm{mol} / \mathrm{l}$, the protein expression of caspase-3 (17 kDa) and Bax in 40 and $80 \mu \mathrm{mol} / 1$ EGCG treatment groups showed an increasing trend that was statistically significant $(\mathrm{P}<0.05)$, displaying a dose- and time-dependent relationship. In contrast, $\mathrm{Bcl}-\mathrm{xL}$ protein expression was higher in the $0 \mu \mathrm{mol} / 1$ group after $24 \mathrm{~h}$ of treatment. With the increase of EGCG concentration to 40 and $80 \mu \mathrm{mol} / \mathrm{l}, \mathrm{Bcl}-\mathrm{xL}$ protein expression gradually decreased and the difference was statistically significant $(\mathrm{P}<0.05)$.

Regulation of Ku70 acetylation and interference with Ku70-Bax interaction by EGCG. Following A549 cell treatment with 0,40 and $80 \mu \mathrm{mol} / 1$ EGCG for 24 and $48 \mathrm{~h}$, co-immunoprecipitation was used to detect the acetylation status of Ku70 and the interaction between Ku70-Bax (Fig. 6). 
A

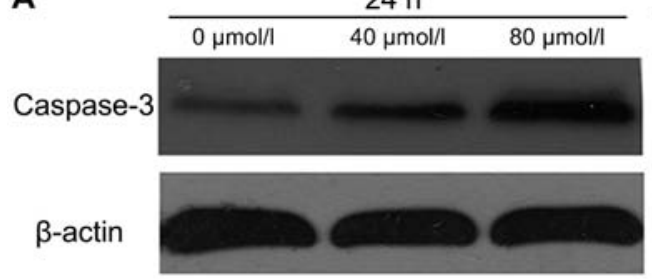

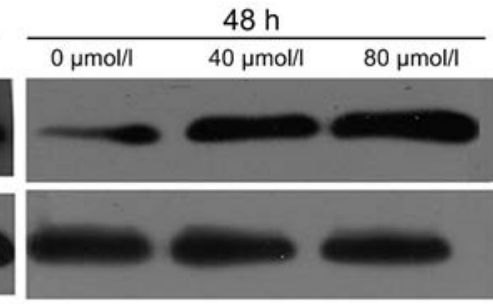

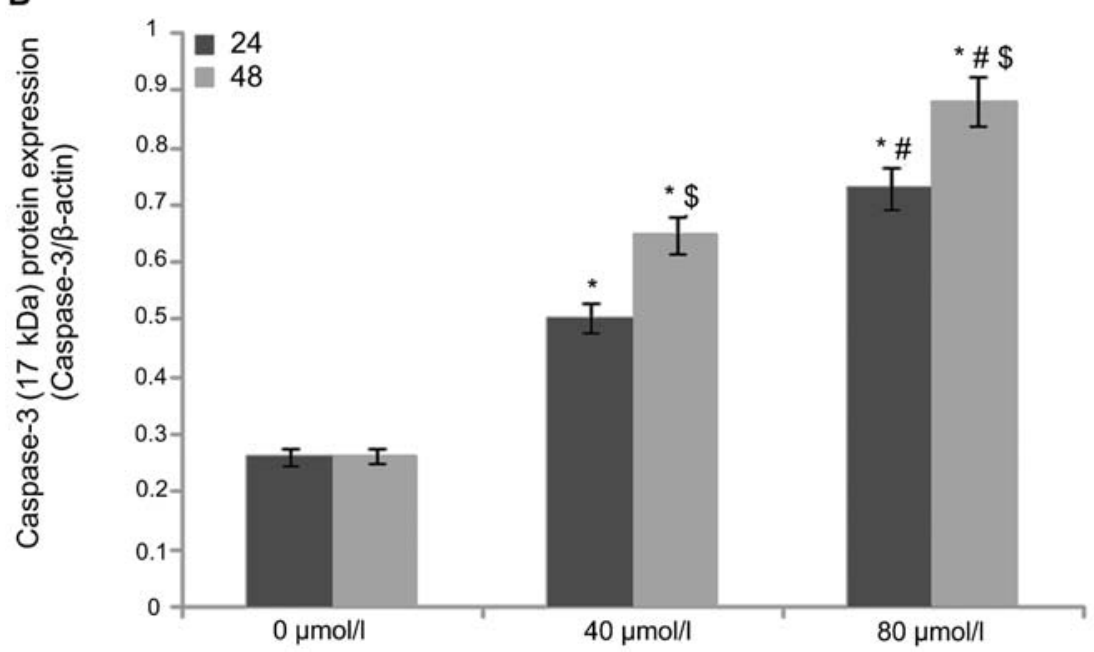

Figure 3. Cleaved caspase-3 expression in A549 cells treated with EGCG. (A) Western blot analysis of cleaved caspase-3 in A549 cells treated with different concentrations of EGCG for different lengths of time. (B) The bands were quantified by densitometry and are represented graphically. The results are the mean of three independent experiments. Using IPP6.0 software (mean $\pm \mathrm{SD}, \mathrm{n}=3$ ). $\mathrm{P}<0.05$, compared with the $0 \mu$ mol $/ 1$ group at the same time; ${ }^{\# \mathrm{P}}<0.05$, compared with the $40 \mu \mathrm{mol} / 1$ group at the same point; ${ }^{\$} \mathrm{P}<0.05$, compared with the same concentration group at $24 \mathrm{~h}$. The protein expression of cleaved caspase-3 in 40 and $80 \mu \mathrm{mol} / 1$ EGCG treatment groups showed an increasing trend dose- and time-dependently.

A

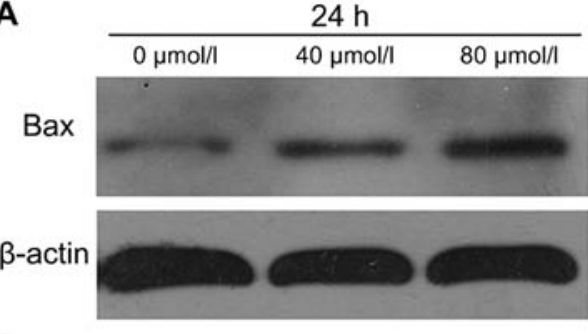

$48 \mathrm{~h}$

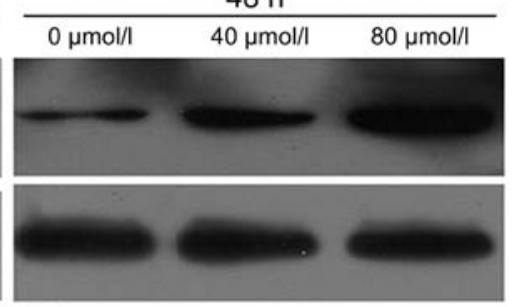

\section{B}

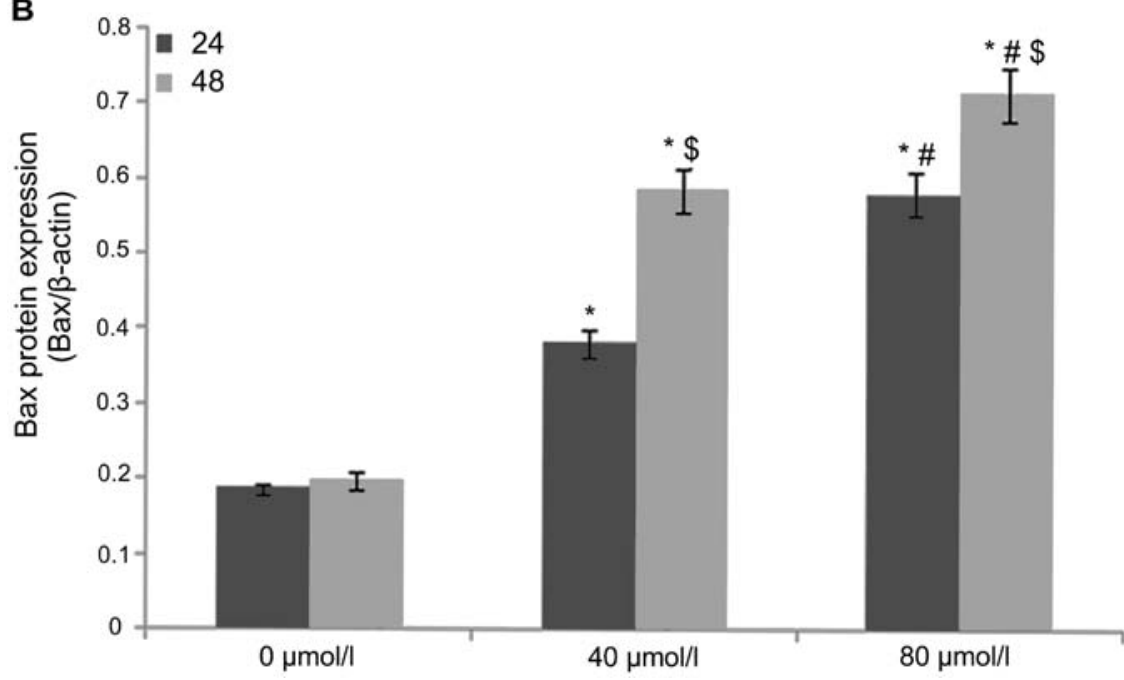

Figure 4. Bax expression in A549 cells treated with EGCG. (A) Western blot analysis of Bax protein expression in A549 cells treated with different concentration of EGCG for different lengths of time. (B) The bands were quantified by densitometry and are represented graphically. The results are the mean of three independent experiments. Using IPP6.0 software (mean $\pm \mathrm{SD}, \mathrm{n}=3$ ). ${ }^{*} \mathrm{P}<0.05$, compared with the $0 \mu \mathrm{mol} / 1$ group at the same time; ${ }^{*} \mathrm{P}<0.05$, compared with the $40 \mu \mathrm{mol} / 1$ group at the same point; ${ }^{\$} \mathrm{P}<0.05$, compared with the same concentration group at $24 \mathrm{~h}$. The protein expression of Bax in 40 and $80 \mu \mathrm{mol} / 1 \mathrm{EGCG}$ treatment groups showed an increasing trend dose- and time-dependently. 
A
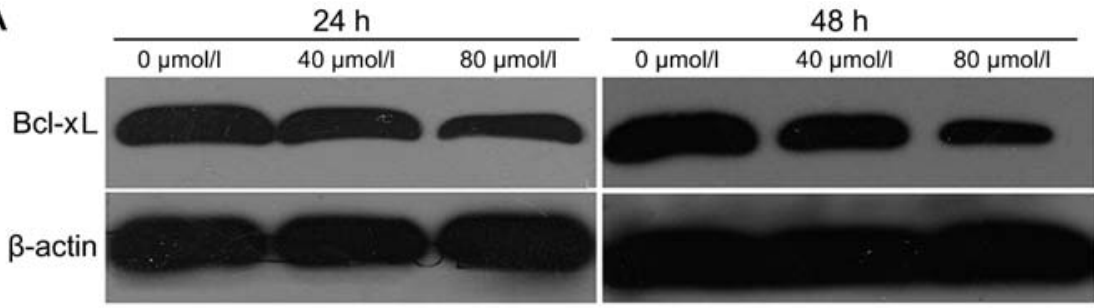

B

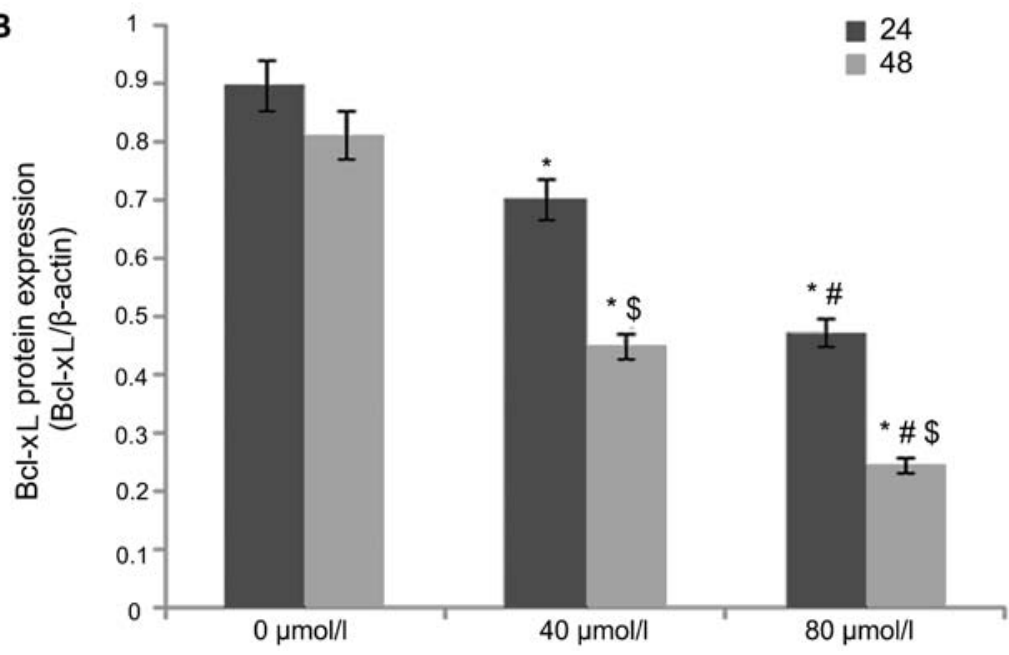

Figure 5. Bcl-xL protein expression in A549 cells treated with EGCG. (A) Western blot analysis of Bcl-xL protein expression in A549 cells treated with different concentrations of EGCG for various lengths of time. (B) The bands were quantified by densitometry and are represented graphically. The results are the mean of three independent experiments. Using IPP6.0 software (mean $\pm \mathrm{SD}, \mathrm{n}=3$ ). ${ }^{*} \mathrm{P}<0.05$, compared with the $0 \mu$ mol/ 1 group at the same time; ${ }^{~} \mathrm{P}<0.05$, compared with the $40 \mu \mathrm{mol} / 1$ group at the same point; ${ }^{\$} \mathrm{P}<0.05$, compared with the same concentration group at $24 \mathrm{~h}$. The protein expression of Bcl-xL in 40 and $80 \mu \mathrm{mol} / \mathrm{l} \mathrm{EGCG} \mathrm{treatment} \mathrm{groups} \mathrm{showed} \mathrm{a} \mathrm{decreasing} \mathrm{trend} \mathrm{dose-} \mathrm{and} \mathrm{time-dependently.}$

A

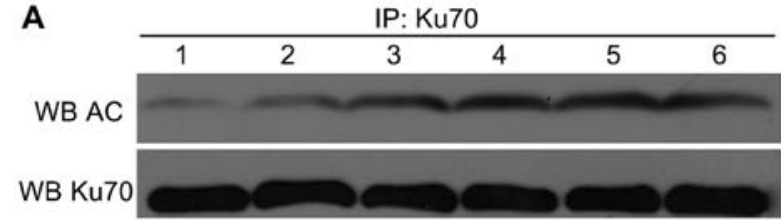

B

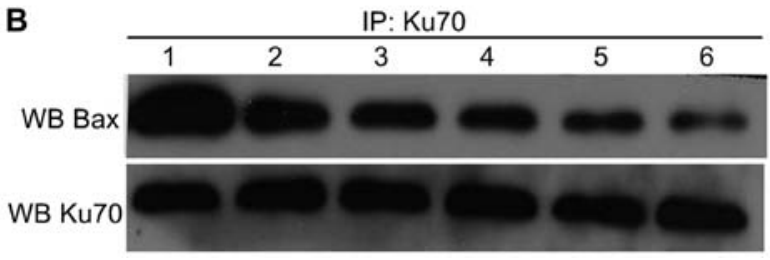

Figure 6. Ku70 acetylation level and Bax-Ku70 protein interaction level in A549 cells treated with EGCG. (A) Ku70 acetylation level in A549 cells treated with different concentrations of EGCG for various lengths of time $(1,0 \mu \mathrm{mol} / 1,24 \mathrm{~h} ; 2,40 \mu \mathrm{mol} / 1,24 \mathrm{~h} ; 3,80 \mu \mathrm{mol} / 1,24 \mathrm{~h} ; 4,0 \mu \mathrm{mol} / 1,48 \mathrm{~h}$; $5,40 \mu \mathrm{mol} / 1,48 \mathrm{~h}$; and $6,80 \mu \mathrm{mol} / 1,48 \mathrm{~h}$ ). (B) Bax-Ku70 protein interaction level in A549 cells treated with different concentrations of EGCG for various lengths of time $(1,0 \mu \mathrm{mol} / 1,24 \mathrm{~h} ; 2,40 \mu \mathrm{mol} / 1,24 \mathrm{~h} ; 3,80 \mu \mathrm{mol} / 1$, $24 \mathrm{~h} ; 4,0 \mu \mathrm{mol} / 1,48 \mathrm{~h} ; 5,40 \mu \mathrm{mol} / 1,48 \mathrm{~h}$; and $6,80 \mu \mathrm{mol} / 1,48 \mathrm{~h}$ ). Co-immunoprecipitation showed Ku70 acetylation status was strengthened, but the interaction of Ku70-Bax was decreased with the EGCG treatment, dose- and time-dependently.

The results showed that in the $0 \mu \mathrm{mol} / \mathrm{l}$ group after treatment for $24 \mathrm{~h}$, the Ku70 acetylation status was weak, but the interaction of Ku70-Bax was strong. With an increase of EGCG concentration to 40 and $80 \mu \mathrm{mol} / 1, \mathrm{Ku} 70$ acetylation status was strengthened, but the interaction of Ku70-Bax showed a decreasing trend. After treatment with the same concentration of EGCG for a different time, the acetylation status of $\mathrm{Ku} 70$ was positively related to the length of treatment. However, the interaction of $\mathrm{Ku} 70$-Bax gradually decreased with the prolongation of treatment.

EGCG induces A549 cell apoptosis with different plasmid transfections. Following A549 cell treatment with different concentrations of EGCG $(0,40$ and $80 \mu \mathrm{M})$ and undergoing plasmid transfections [pCDNA3.1(+), and pCDNA3.1(+)-Ku70 and pCDNA3.1(+)-Ku70 539/542R plasmid transfection groups] for $48 \mathrm{~h}$, Annexin V/PI double staining was used to detect the apoptosis rate of the cells (Fig. 7). The results showed that the early apoptosis rate of A549 cells increased with an increasing concentration of EGCG in the pCDNA3.1(+) plasmid transfection group. However, the early cell apoptosis rate was similar with or without EGCG treatment in the pCDNA3.1(+)-Ku70 and in the pCDNA3.1(+)-Ku70 $0^{539 / 542 \mathrm{R}}$ plasmid transfection groups. In contrast, for cells treated with EGCG, the early apoptosis rate in the pCDNA3.1(+)-Ku70 and in the pCDNA3.1(+)-Ku70 $0^{539 / 542 \mathrm{R}}$ plasmid transfection groups was lower than that in the control group and in the pCDNA3.1(+) plasmid transfection group $(\mathrm{P}<0.05)$.

Effects of EGCG on cleaved caspase-3 and Bax expression in A549 cells with different plasmid transfections. A549 cells were treated with different concentrations of EGCG $(0,40$ and $80 \mu \mathrm{mol} / \mathrm{l})$ and undergwent different plasmid transfections [control,pCDNA3.1(+),pCDNA3.1(+)-Ku70 and pCDNA3.1(+)$\mathrm{Ku} 70^{539 / 542 \mathrm{R}}$ plasmid transfection groups] for $48 \mathrm{~h}$, western blot 
A
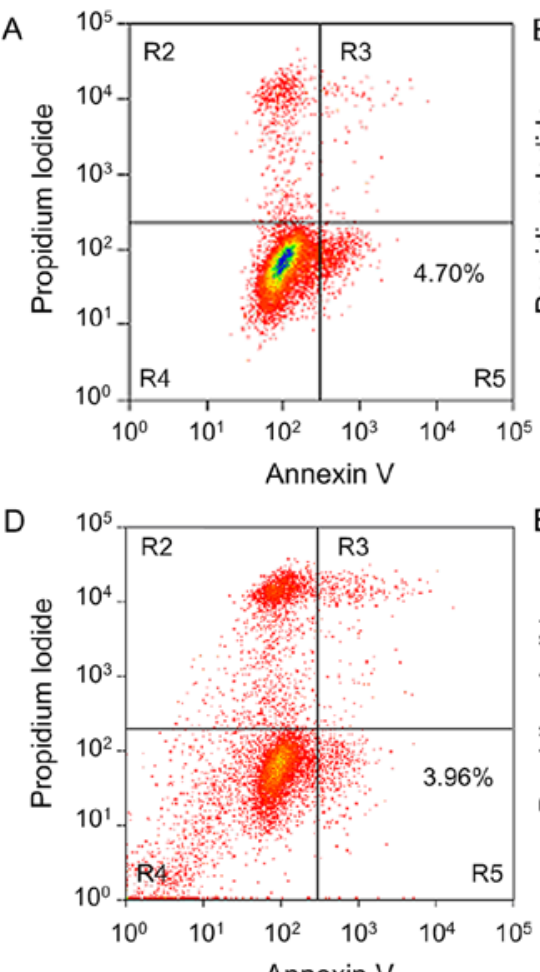

Annexin $\mathrm{V}$

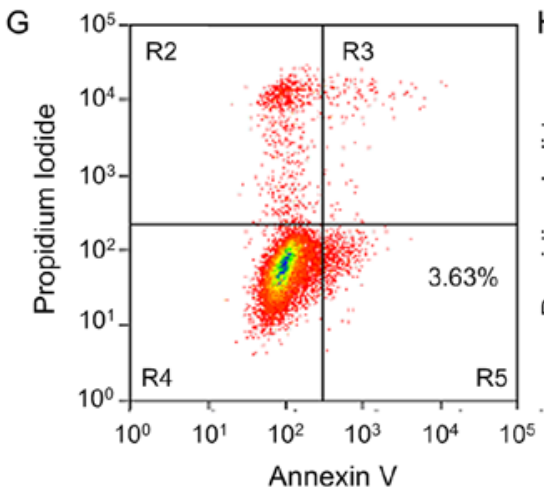

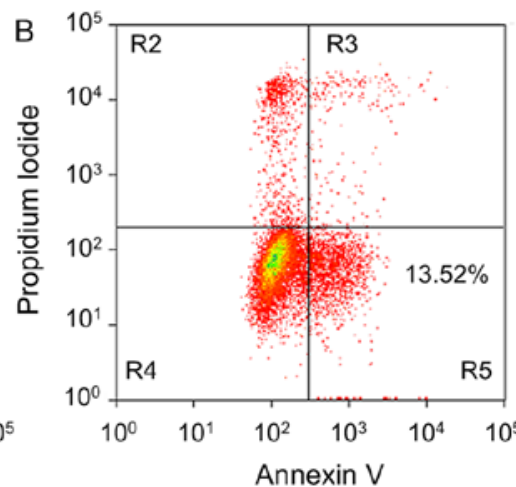
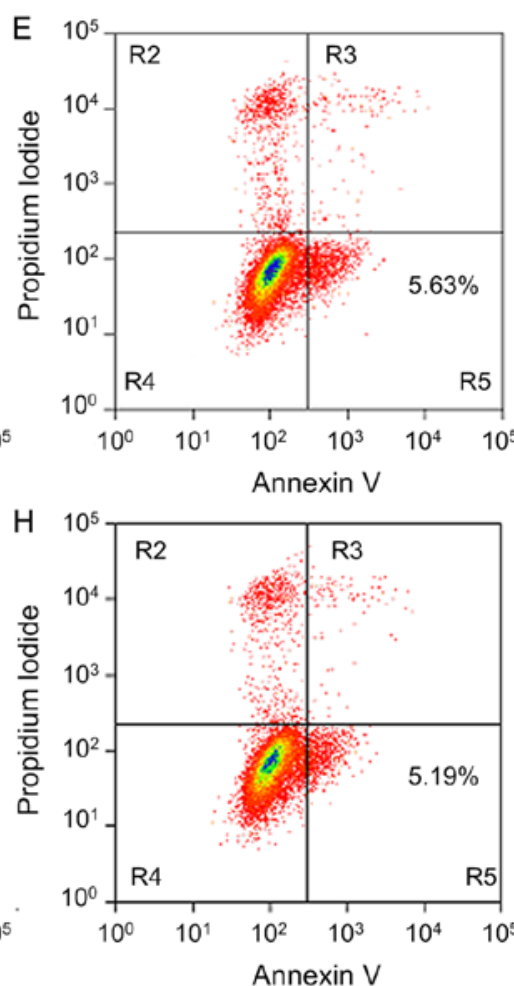
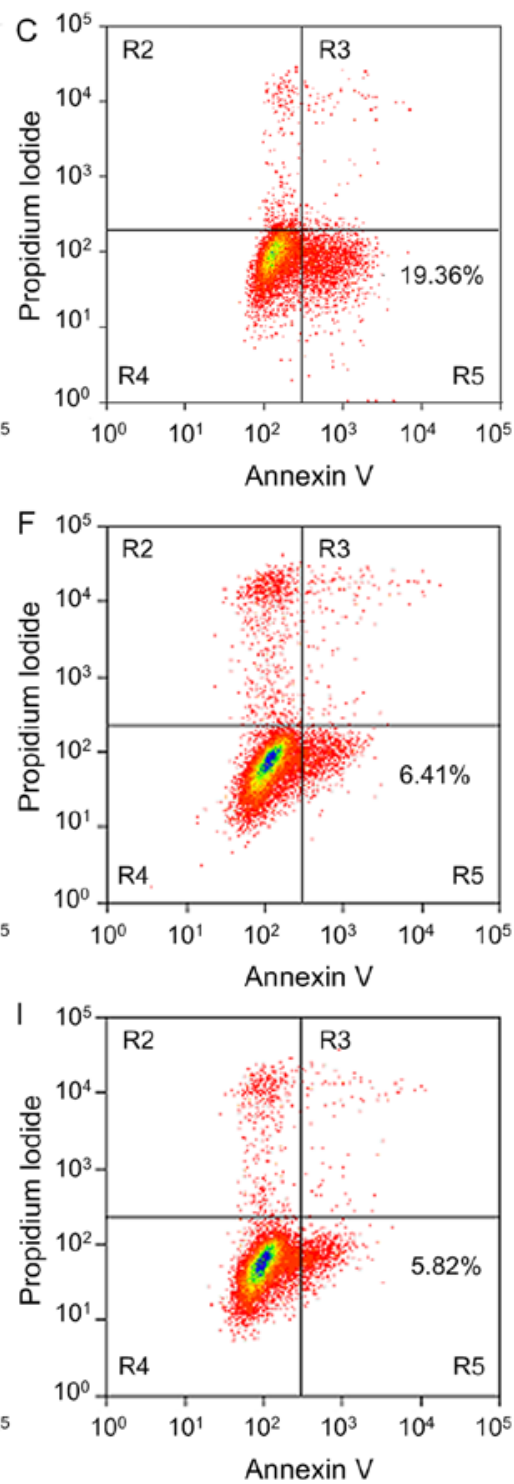

Figure 7. Apoptosis inducing effect of A549 cells treated with EGCG, after undergoing different plasmid transfections for 48 h, detected by Annexin V/PI double staining. (A) pCDNA3.1(+) plasmid transfection group, $0 \mu \mathrm{mol} / 1$; (B) pCDNA3.1(+) plasmid transfection group, $40 \mu \mathrm{mol} / 1$; (C) pCDNA3.1(+) plasmid transfection group, $80 \mu \mathrm{mol} / 1$; (D) pCDNA3.1(+)-Ku70 plasmid transfection group, $0 \mu \mathrm{mol} / 1$; (E) pCDNA3.1(+)-Ku70 plasmid transfection group, $40 \mu \mathrm{mol} / 1$; (F) pCDNA3.1(+)-Ku70 plasmid transfection group, $80 \mu \mathrm{mol} / 1$; (G) pCDNA3.1 (+)-Ku70 ${ }^{539 / 542 \mathrm{R}}$ plasmid transfection group, $0 \mu \mathrm{mol} / 1$; (H) pCDNA3.1 $(+)-\mathrm{Ku} 70^{539 / 542 \mathrm{R}}$ plasmid transfection group, $40 \mu \mathrm{mol} / \mathrm{l}$; (I) pCDNA3.1 (+)-Ku70 $539 / 542 \mathrm{R}$ plasmid transfection group, $80 \mu \mathrm{mol} / 1$. For cells treated with EGCG, the early apoptosis rate in the pCDNA3.1(+)-Ku70 and in the pCDNA3.1(+)-Ku70 ${ }^{539 / 542 R}$ plasmid transfection groups was lower than that in the control and in the pCDNA3.1(+) plasmid transfection groups.

analysis was used to detect the protein expression of cleaved caspase-3 (Fig. 8) and Bax (Fig. 9). Caspase-3 (17 kDa) expression in the pCDNA3.1(+)-Ku70 plasmid transfection group and in the pCDNA3.1(+)-Ku70 $0^{539 / 542 \mathrm{R}}$ plasmid transfection group was similar to that of the control group and the pCDNA3.1(+) plasmid transfection group without EGCG treatment. In contrast, for cells treated with EGCG, caspase-3 (17 kDa) expression in the pCDNA3.1(+)-Ku70 and in the pCDNA3.1(+)-Ku70 ${ }^{539 / 542 \mathrm{R}}$ plasmid transfection groups was lower than that of the control and the pCDNA3.1(+) plasmid transfection groups. In particular, caspase-3 expression in the pCDNA3.1(+)-Ku70 ${ }^{539 / 542 \mathrm{R}}$ plasmid transfection group was lower, and the difference was statistically significant $(\mathrm{P}<0.05)$. However, the Bax protein expression in A549 cells showed no significant difference after intervention by different plasmid transfections with the same concentration of EGCG.
Effect of EGCG on the Bax-Ku70 interaction in A549 cells with different plasmid transfections. A549 cells were treated with different concentrations of EGCG (0, 40 and $80 \mu \mathrm{mol} / \mathrm{l})$ then underwent different plasmid transfections [control, pCDNA3.1(+), pCDNA3.1(+)-Ku70 and pCDNA3.1 $(+)-\mathrm{Ku} 70^{539 / 542 \mathrm{R}}$ plasmid transfection groups] for $48 \mathrm{~h}$, co-immunoprecipitation was used to detect the interaction of Ku70-Bax (Fig. 10). The interaction of Bax-Ku70 in the pCDNA3.1(+)-Ku70 and in the pCDNA3.1(+)-Ku70 $0399 / 542 \mathrm{R}$ plasmid transfection groups was similar to that of the control group and the pCDNA3.1(+) plasmid transfection group without EGCG treatment. In contrast, for cells treated with EGCG, the interaction of Bax-Ku70 in the pCDNA3.1(+)-Ku70 and in the pCDNA3.1(+)-Ku70 ${ }^{539 / 542 \mathrm{R}}$ plasmid transfection groups was significantly stronger than that of the control and the pCDNA3.1(+) plasmid transfection groups. In 
A

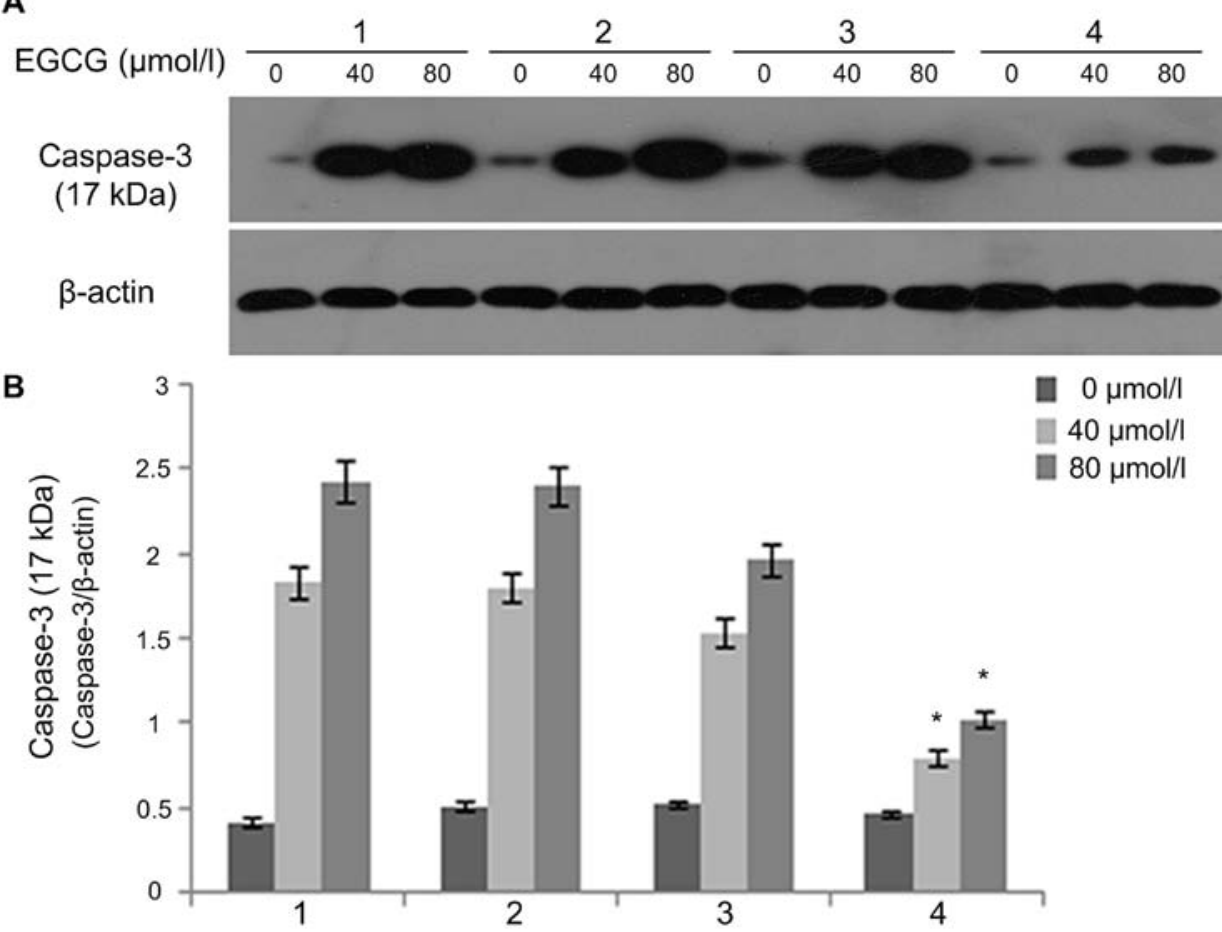

Figure 8. Cleaved caspase-3 expression in A549 cells treated with EGCG after different plasmid transfections at 48 h [1, control group; 2, pCDNA3.1(+) plasmid transfection group; 3, pCDNA3.1(+)-Ku70 plasmid transfection group; and 4, pCDNA3.1 (+)-Ku70 ${ }^{539 / 542 R}$ plasmid transfection group control group]. (A) Western blot analysis of cleaved caspase-3 in A549 cells treated with different concentrations of EGCG after different plasmid transfections at 48 h (B) The bands were quantified by densitometry and are represented graphically. The results are the mean of three independent experiments. Using IPP6.0 software (mean $\pm \mathrm{SD}, \mathrm{n}=3$ ). ${ }^{*} \mathrm{P}<0.05$, compared with the control group treated by the same concentration of EGCG. With the same concentration of EGCG treatment, the cleaved caspase expression in the pCDNA3.1(+)-Ku70 and in the pCDNA3.1(+)-Ku70 $0^{539 / 542 R}$ plasmid transfection groups was lower than that of the control and the pCDNA3.1(+) plasmid transfection groups, but the difference was statistically significant only in the pCDNA3.1(+)-Ku70 ${ }^{539 / 542 R}$ plasmid transfection group.

A

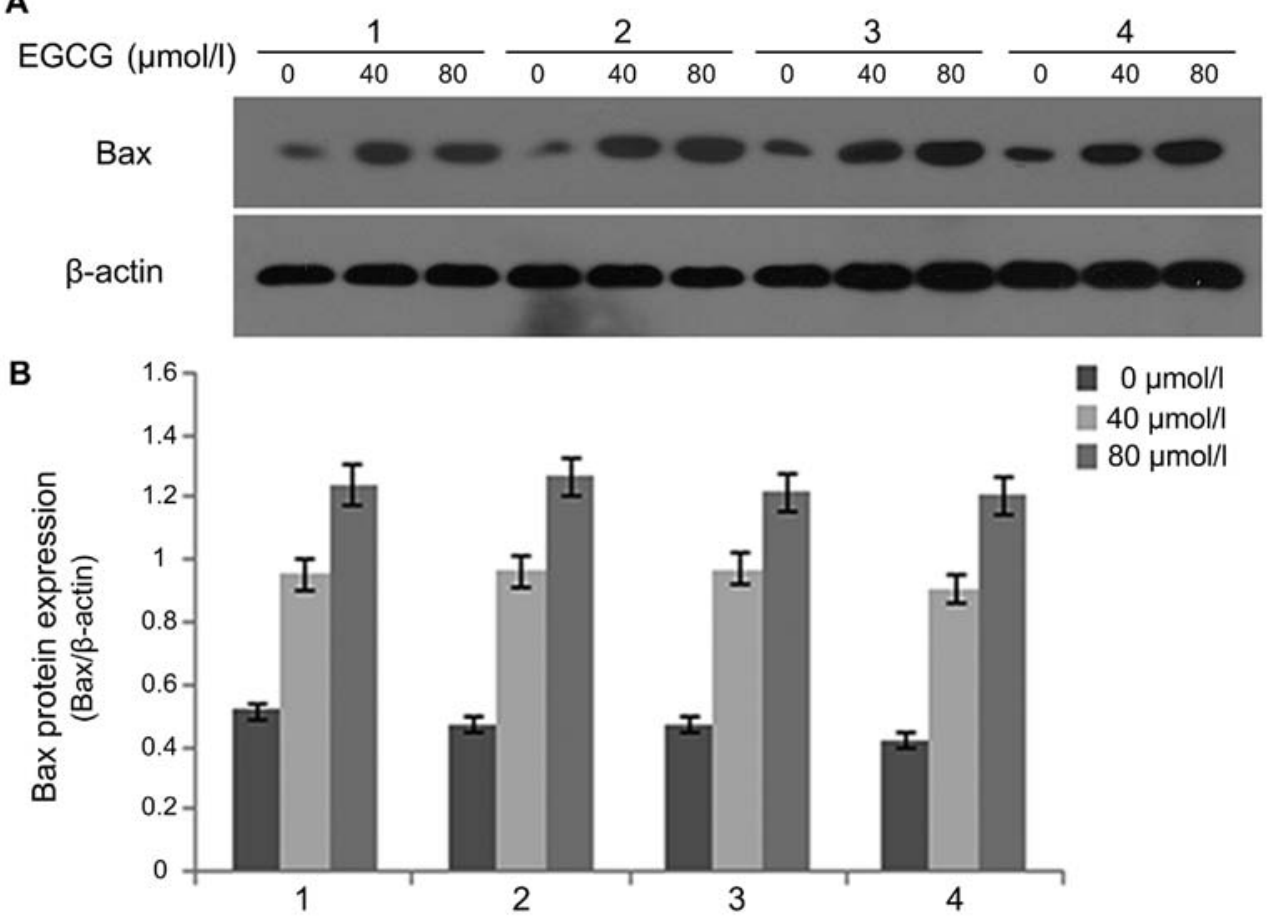

Figure 9. Bax protein expression in A549 cells treated with EGCG after different plasmid transfections for $48 \mathrm{~h}$ [1, control group; 2, pCDNA3.1(+) plasmid transfection group; 3, pCDNA3.1(+)-Ku70 plasmid transfection group; and 4, pCDNA3.1 (+)-Ku70 $0^{539 / 542 R}$ plasmid transfection group control group]. (A) Western blot analysis of Bax protein expression in A549 cells treated with different concentrations of EGCG after various plasmid transfections at $48 \mathrm{~h}$. (B) The bands were quantified by densitometry and are presented graphically. The results are the mean of three independent experiments. Using IPP6.0 software (mean \pm SD, $\mathrm{n}=3$ ). The Bax protein expression in A549 cells showed no significant difference after intervention by different plasmid transfections with the same concentration of EGCG treatment. 
IP:Ku70

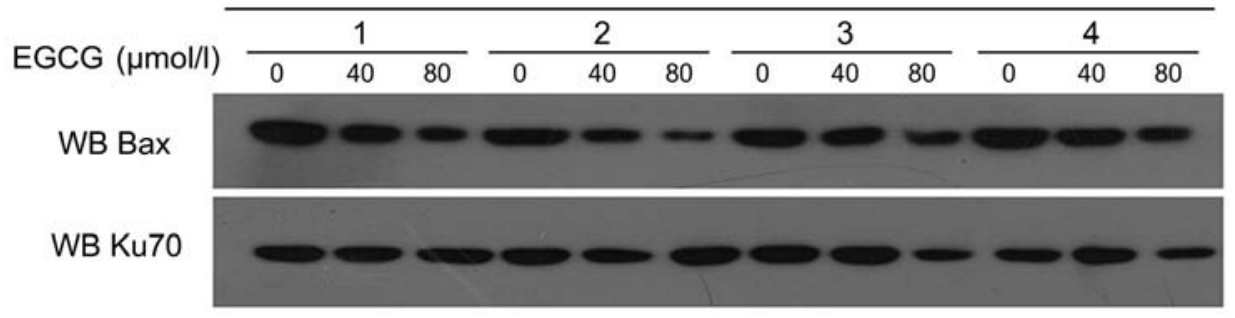

Figure 10. Bax-Ku70 protein interaction level in A549 cells treated with EGCG after different plasmid transfections for 48 h [1, control group; 2, pCDNA3.1(+) plasmid transfection group; 3, pCDNA3.1(+)-Ku70 plasmid transfection group; and 4, pCDNA3.1 (+)-Ku70 $0^{539 / 542 R}$ plasmid transfection group control group]. With the same concentration of EGCG treatment, the interaction of Bax-Ku70 in the pCDNA3.1(+)-Ku70 and in the pCDNA3.1(+)-Ku70 ${ }^{539 / 542 R}$ plasmid transfection groups was lower than that of the control and the pCDNA3.1(+) plasmid transfection groups, but the difference was statistically significant only in the pCDNA3.1(+)-Ku70 $0^{539 / 542 R}$ plasmid transfection group.

particular, the interaction of Bax-Ku70 was stronger in the pCDNA3.1(+)-Ku70 $539 / 542 \mathrm{R}$ plasmid transfection group, and the difference was statistically significant $(\mathrm{P}<0.05)$.

\section{Discussion}

Green tea is one of the most consumed beverages worldwide, particularly in Asian countries. EGCG is the main monomer component of green tea polyphenols. Many research studies have shown that EGCG has an inhibitory effect on the occurrence and development of malignant tumors. The present study showed that EGCG could effectively induce apoptosis of human lung adenocarcinoma A549 cells, further confirming our previous results. The outstanding advantage of EGCG is the milder effect on normal cells while killing tumor cells. Kang et al showed that the killing effect of EGCG on Ewing's sarcoma cells of children was stronger than that on normal cells (23). In their study, 25 and $50 \mu \mathrm{mol} / 1$ EGCG showed obvious cell growth inhibition on Ewing's sarcoma cell lines TC32 and TC71, respectively, but only mild damage on the normal human microvascular endothelial cell line HBMEC, up to an EGCG concentration of $100 \mu \mathrm{mol} / \mathrm{l}$. A similar drug action was also shown by EGCG on the adrenal carcinoma cell line NCI-H295 and normal primary human embryonic skin cells (24).

Apoptosis is a complex process regulated by several molecules that function as either promoters, including Bax, Bak and caspases, or inhibitors of the cell death process such as Bcl-2, Bcl-xL and the IAP proteins (25). Studies have shown that EGCG can effectively regulate various key molecules in cell mitochondrial apoptosis pathways in other tumors as a potential antitumor substance $(18,19)$. Our previous study showed that the long-term ingestion of green tea could effectively prevent lung cancer, in rats, induced by the carcinogen 3,4-benzopyrene $(\mathrm{B}[\mathrm{a}] \mathrm{P})$, a process that may be related to the EGCG-mediated upregulation of P53 expression and downregulation of Bcl-2 expression (26). The present study showed that on one hand, EGCG upregulated the expression of the apoptosis-promoting factor Bax, whereas on the other hand, it downregulated the expression of the apoptosis inhibitor Bcl-xL. EGCG also activated a member of the caspase-3 family to achieve anticancer effects, suggesting that EGCG may regulate human lung adenocarcinoma A549 cells through the mitochondrial apoptosis pathway. Hastak et al knocked down Bax, and then the wild-type group and the Bax interference group were treated with placebo and EGCG. The results showed that the apoptosis rate in the Bax interference group was significantly higher than that of the wild-type group after treatment with EGCG. However, there was no obvious difference in the apoptosis rate between the Bax interference group and the wild-type group after treatment with placebo, suggesting that EGCG-induced cell apoptosis required the interference of Bax (27).

Sawada et al screened functional inhibitory protein $\mathrm{Ku} 70$ combined with Bax by the functional screening method based on yeast and confirmed that $\mathrm{Ku} 70$ had a direct inhibitory effect on the Bax-mediated mitochondrial apoptosis pathway (28). Further studies showed that the inhibitory effect was related to the direct binding of BIP and Bax on the $\mathrm{Ku} 70$ protein $\mathrm{C}$ terminal (29). The present study also showed that $\mathrm{Ku} 70$ and its acetylation status may play a critical role in the mitochondrial apoptosis pathway mediated by Bax. Our study showed that for A549 cells under the influence of EGCG, the interaction of Bax-Ku70 decreased with an increasing concentration of EGCG. In addition, their interaction showed a decreasing trend with prolongation of the action time. To further understand whether the EGCG-mediated interaction of Ku70-Bax was related to $\mathrm{Ku} 70$ acetylation, this study further detected the regulation of EGCG on the Ku70 acetylation status of A549 cells. The results showed that EGCG could also effectively upregulate the acetylation status of A549 cells and showed that this effect was concentration- and time-dependent.

Cohen et al (30) compared the Ku70 amino acid sequence and the amino acid sequence of other factors regulated by acetylation, including P53, FEN1, GATA1 and EFIIL $\beta$. The results showed that the 530-583 sequence of $\mathrm{Ku} 70$ was similar to the amino acid sequence in the acetylation area of the above factors. A point mutation of several lysine residues in the 530-583 sequence of Ku70 was introduced. The results revealed that if lysine was mutated into arginine, the loci would lose the acetylation function but would not affect the entire $\mathrm{Ku} 70$ protein function. The final results showed that two loci, K539 and K542, played a crucial role in Ku70 acetylation (31). Therefore, in this study, the pCDNA3.1(+)-Ku70 and pCDNA3.1(+)-Ku70 ${ }^{539 / 542 \mathrm{R}}$ plasmids were constructed and successfully transferred into A549 cells. The two acetylation loci of Ku70, K539 and K542, were lost after pCDNA3.1(+)$\mathrm{Ku} 70^{539 / 542 \mathrm{R}}$ transfection, and therefore, the acetylation 
function was lost. Thereafter, we determined that the apoptosis-promoting effect of EGCG on A549 cells was obviously weakened, concurrent with strengthening of the Bax-Ku70 interaction and a decline in cleaved caspase-3 expression, after pCDNA3.1(+)-Ku70 plasmid and pCDNA3.1(+)-Ku70 ${ }^{539 / 542 R}$ plasmid transfection. This result verified that EGCG may induce apoptosis of human lung adenocarcinoma A549 cells through the K539 and K542 acetylation loci of Ku70.

\section{Acknowledgements}

The present study was supported by the National Key Scientific and Technology Support Program. Collaborative innovation of Clinical Research for chronic obstructive pulmonary disease and lung cancer, no. 2013BAI09B09.

\section{References}

1. Jemal A, Bray F, Center MM, Ferlay J, Ward E and Forman D: Global cancer statistics. CA Cancer J Clin 61: 69-90, 2011.

2. Kushi LH, Doyle C, McCullough M, Rock CL, DemarkWahnefried W, Bandera EV, Gapstur S, Patel AV, Andrews K and Gansler T; American Cancer Society 2010 Nutrition and Physical Activity Guidelines Advisory Committee: American Cancer Society Guidelines on nutrition and physical activity for cancer prevention: Reducing the risk of cancer with healthy food choices and physical activity. CA Cancer J Clin 62: 30-67, 2012.

3. Jin L, Li C, Xu Y, Wang L, Liu J, Wang D, Hong C, Jiang Z, Ma Y, Chen Q, et al: Epigallocatechin gallate promotes p53 accumulation and activity via the inhibition of MDM2-mediated p53 ubiquitination in human lung cancer cells. Oncol Rep 29: 1983-1990, 2013.

4. Ma YC, Li C, Gao F, Xu Y, Jiang ZB, Liu JX and Jin LY: Epigallocatechin gallate inhibits the growth of human lung cancer by directly targeting the EGFR signaling pathway. Oncol Rep 31: 1343-1349, 2014.

5. Lee YH, Kwak J, Choi HK, Choi KC, Kim S, Lee J, Jun W, Park HJ and Yoon HG: EGCG suppresses prostate cancer cell growth modulating acetylation of androgen receptor by antihistone acetyltransferase activity. Int J Mol Med 30: 69-74, 2012.

6. Tang Y,Zhao DY, Elliott S, Zhao W, Curiel TJ, Beckman BS and Burow ME: Epigallocatechin-3 gallate induces growth inhibition and apoptosis in human breast cancer cells through survivin suppression. Int J Oncol 31: 705-711, 2007.

7. Yang CS and Wang ZY: Tea and cancer. J Natl Cancer Inst 85: 1038-1049, 1993

8. Wu H, Xin Y, Xiao Y and Zhao J: Low-dose docetaxel combined with (-)-epigallocatechin-3-gallate inhibits angiogenesis and tumor growth in nude mice with gastric cancer xenografts. Cancer Biother Radiopharm 27: 204-209, 2012.

9. Zhang G, Wang Y, Zhang Y, Wan X, Li J, Liu K, Wang F, Liu K, Liu Q, Yang C, et al: Anti-cancer activities of tea epigallocatechin-3-gallate in breast cancer patients under radiotherapy. Curr Mol Med 12: 163-176, 2012.

10. Tudoran O, Soritau O, Balacescu O, Balacescu L, Braicu C, Rus M, Gherman C, Virag P, Irimie F and Berindan-Neagoe I: Early transcriptional pattern of angiogenesis induced by EGCG treatment in cervical tumour cells. J Cell Mol Med 16: 520-530, 2012.

11. Lu YP, Lou YR, Xie JG, Peng QY, Liao J, Yang CS, Huang MT and Conney AH: Topical applications of caffeine or (-)-epigallocatechin gallate (EGCG) inhibit carcinogenesis and selectively increase apoptosis in UVB-induced skin tumors in mice. Proc Natl Acad Sci USA 99: 12455-12460, 2002.

12. Syed DN, Afaq F, Kweon MH, Hadi N, Bhatia N, Spiegelman VS and Mukhtar H: Green tea polyphenol EGCG suppresses cigarette smoke condensate-induced NF-kappaB activation in normal human bronchial epithelial cells. Oncogene 26: 673-682, 2007.
13. Lee MH, Han DW, Hyon SH and Park JC: Apoptosis of human fibrosarcoma HT-1080 cells by epigallocatechin-3- $O$-gallate via induction of $\mathrm{p} 53$ and caspases as well as suppression of $\mathrm{Bcl}-2$ and phosphorylated nuclear factor- $\mathrm{KB}$. Apoptosis 16: 75-85, 2011.

14. Tsukamoto S, Hirotsu K, Kumazoe M, Goto Y, Sugihara K, Suda T, Tsurudome Y, Suzuki T, Yamashita S, Kim Y, et al: Green tea polyphenol EGCG induces lipid-raft clustering and apoptotic cell death by activating protein kinase $C \delta$ and acid sphingomyelinase through a $67 \mathrm{kDa}$ laminin receptor in multiple myeloma cells. Biochem J 443: 525-534, 2012.

15. Adachi S, Nagao T, Ingolfsson HI, Maxfield FR, Andersen OS, Kopelovich L and Weinstein IB: The inhibitory effect of (-)-epigallocatechin gallate on activation of the epidermal growth factor receptor is associated with altered lipid order in HT29 colon cancer cells. Cancer Res 67: 6493-6501, 2007.

16. Wei LH, Kuo ml, Chen CA, Chou CH, Lai KB, Lee CN and Hsieh CY: Interleukin-6 promotes cervical tumor growth by VEGF-dependent angiogenesis via a STAT3 pathway. Oncogene 22: 1517-1527, 2003.

17. Wang X, Hao MW, Dong K, Lin F, Ren JH and Zhang HZ: Apoptosis induction effects of EGCG in laryngeal squamous cell carcinoma cells through telomerase repression. Arch Pharm Res 32: 1263-1269, 2009

18. Wang J, Xie Y, Feng Y, Zhang L, Huang X, Shen X and Luo X: (-)-Epigallocatechingallate induces apoptosis in B lymphoma cells via caspase-dependent pathway and $\mathrm{Bcl}-2$ family protein modulation. Int J Oncol 46: 1507-1515, 2015.

19. Sonoda JI, Ikeda R, Baba Y, Narumi K, Kawachi A, Tomishige E, Nishihara K, Takeda Y, Yamada K, Sato K, et al: Green tea catechin, epigallocatechin-3-gallate, attenuates the cell viability of human non-small-cell lung cancer A549 cells via reducing Bcl-xL expression. Exp Ther Med 8: 59-63, 2014.

20. Hurwitz JL, Stasik I, Kerr EM, Holohan C, Redmond KM, McLaughlin KM, Busacca S, Barbone D, Broaddus VC, Gray SG, et al: Vorinostat/SAHA-induced apoptosis in malignant mesothelioma is FLIP/caspase 8-dependent and HR23B-independent. Eur J Cancer 48: 1096-1107, 2012.

21. Hada M and Kwok RP: Regulation of ku70-bax complex in cells. J Cell Death 7: 11-13, 2014.

22. Li JJ, Gu QH, Li M, Yang HP, Cao LM and Hu CP: Role of Ku70 and Bax in epigallocatechin-3-gallate-induced apoptosis of A549 cells in vivo. Oncol Lett 5: 101-106, 2013.

23. Kang HG, Jenabi JM, Liu XF, Reynolds CP, Triche TJ and Sorensen PH: Inhibition of the insulin-like growth factor I receptor by epigallocatechin gallate blocks proliferation and induces the death of Ewing tumor cells. Mol Cancer Ther 9: 1396-1407, 2010

24. Wu PP, Kuo SC, Huang WW, Yang JS, Lai KC, Chen HJ, Lin KL, Chiu YJ, Huang LJ and Chung JG: (-)-Epigallocatechin gallate induced apoptosis in human adrenal cancer NCI-H295 cells through caspase-dependent and caspase-independent pathway. Anticancer Res 29: 1435-1442, 2009.

25. Deveraux QL, Schendel SL and Reed JC: Antiapoptotic proteins. The bcl-2 and inhibitor of apoptosis protein families. Cardiol Clin 19: 57-74, 2001

26. Gu Q, Hu C, Chen Q and Xia Y: Tea polyphenols prevent lung from preneoplastic lesions and effect p53 and bcl-2 gene expression in rat lung tissues. Int J Clin Exp Pathol 6: 15231531,2013

27. Hastak K, Agarwal MK, Mukhtar H and Agarwal ML: Ablation of either $\mathrm{p} 21$ or Bax prevents p53-dependent apoptosis induced by green tea polyphenol epigallocatechin-3-gallate. FASEB J 19: 789-791, 2005

28. Sawada M, Sun W, Hayes P, Leskov K, Boothman DA and Matsuyama S: Ku70 suppresses the apoptotic translocation of Bax to mitochondria. Nat Cell Biol 5: 320-329, 2003.

29. Sawada M, Hayes P and Matsuyama S: Cytoprotective membrane-permeable peptides designed from the Bax-binding domain of Ku70. Nat Cell Biol 5: 352-357, 2003.

30. Cohen HY, Lavu S, Bitterman KJ, Hekking B, Imahiyerobo TA, Miller C, Frye R, Ploegh H, Kessler BM and Sinclair DA: Acetylation of the $\mathrm{C}$ terminus of $\mathrm{Ku} 70$ by $\mathrm{CBP}$ and PCAF controls Bax-mediated apoptosis. Mol Cell 13: 627-638, 2004. 